\title{
Computasseia: Um Jogo para o Ensino de História da Computação
}

\author{
Jean Carlos Oliveira Santos, Karen da Silva Figueiredo \\ Instituto de Computação - Universidade Federal do Mato Grosso (UFMT) \\ Cuiabá, Mato Grosso, Brasil \\ jcoliveira93egmail.com, karen@ic.ufmt.br
}

\begin{abstract}
The study of History of Computing is necessary to develop the knowledge about the essentials of the area; to understand the evolution of the resources, current techniques and methods; and to expand the critical thinking regarding the present and future of Computing. However, the process of learning the History of Computing can become very boring because of the many names, facts and dates involved. The use of games as educational resources to support computer education is a strategy which can change a boring experience into an engaging experience. This paper proposes a strategy card game about History of Computing that can be used at different levels of education.
\end{abstract}

Resumo. Estudar a história da Computação é essencial para conhecer os fundamentos da área, entender a evolução dos recursos, técnicas e métodos atuais e ampliar o senso crítico com relação ao desenvolvimento da Computação no presente e futuro. Entretanto, o ensino de História da Computação pode tornar-se enfadonho, com a presença de muitos nomes, fatos e datas. A utilização de jogos como recursos educacionais no apoio do ensino de Computação é uma estratégia capaz de transformar uma experiência entediante em uma experiência engajadora. Este trabalho propõe um jogo de cartas sobre diversos conteúdos relacionados à história da Computação que pode ser utilizado em diferentes níveis de ensino.

\section{Introdução}

É crescente o reconhecimento nas universidades de que estudantes de Computação (Ciência da Computação, Sistemas de Informação, entre outros cursos) devem compreender, não somente os aspectos técnicos, mas também os aspectos culturais da sua área para a evolução dos seus estudos acadêmicos [Berry et al. 2011].

Uma grande contribuição neste sentido é o estudo da história da Computação. Diversas universidades possuem em seus currículos dos cursos de Computação alguma disciplina que discute os aspectos históricos da área como parte total ou parcial da ementa. Segundo Fonseca Filho (2007), a Universidade de Stanford e o Instituto Charles Babbage da Universidade de Manchester podem ser citados como algumas das instituições pioneiras na criação das disciplinas de História da Computação na década de 1990.

Estudar a história da Computação é essencial para conhecer os fundamentos da 
área, entender a evolução dos recursos, técnicas e métodos utilizados atualmente e ampliar o senso crítico com relação ao desenvolvimento da Computação no presente e suas perspectivas futuras. Entretanto, o ensino de história da Computação possui, em certos aspectos, as mesmas características do ensino de uma disciplina típica de História. Dependendo da estratégia pedagógica utilizada, a disciplina pode ser considerada entediante e de propósito duvidoso ou relevante, inspiradora $\mathrm{e}$ recompensadora para os alunos [Haydn et al. 2014].

Entre as estratégias contemporâneas para um ensino mais atraente e engajador encontra-se a utilização de jogos sérios, do inglês "serious games", produtos desenvolvidos com princípios de design de jogo interativo, com o objetivo de transmitir um conteúdo educacional ou de treinamento ao usuário que vai além do entretenimento, i.e. que tenham uma mensagem a transmitir [Michael e Chen 2005]. Os jogos sérios estão sendo empregados com sucesso tanto no ensino de disciplinas de História e.g. [Baptista e Carvalho 2013, Froschauer et al. 2011, Neves 2011], como em diversas iniciativas em disciplinas da área de Computação, tais como Algoritmos e Estrutura de Dados [Nunes e Parreira Júnior 2015, Vahldick et al. 2015], Redes [Krassmann et al. 2015] e Teoria da Computação [Leite et al. 2014].

O objetivo deste trabalho é propor um jogo sério para o ensino de História da Computação chamado Computasseia. O Computasseia é um jogo de cartas de estratégia sobre diversos conteúdos relacionados à história da Computação que pode ser utilizado em diferentes níveis de ensino (técnico, graduação e pós-graduação) a fim de auxiliar na retenção de conteúdos e no engajamento com a disciplina. A partir desta introdução, este trabalho está organizado da seguinte forma: a importância do ensino da História da Computação é discutida na Seção 2; a Seção 3 descreve alguns trabalhos relacionados; o design do jogo Computasseia, seus elementos e dinâmica são apresentados na Seção 4; e por fim, as conclusões e trabalhos futuros são traçados na Seção 5.

\section{Por que aprender História da Computação?}

Fonseca Filho (2007) descreve no único livro de língua portuguesa brasileira (disponível até o presente momento) totalmente dedicado ao assunto de História da Computação, cinco motivações para se estudar História da Computação, são essas:

- Necessidade de discernir fundamentos: o ensino de História da Computação é fundamental para compreender que os eventos não estão individualizados, que por muitas vezes são complexos e que influenciam significativamente o saber atual. É preciso possuir um quadro que os reúna para ter-se uma ideia geral, coerente e significativa.

- Incentivar à educação para a qualidade do software: o estudo da História da Computação sob a ótica das ideias e conceitos que formaram a base do desenvolvimento da Computação pode contribuir para sensibilizar e entusiasmar o aluno sobre a importância dos fundamentos teóricos, e o desenvolvimento de software e tecnologia de qualidade.

- Tornar claros e ligar os fatos: é possível compreender a História da Computação como uma forma de olhar para trás com o intuito de descobrir paralelismos e analogias com a tecnologia moderna e assim, proporcionar uma 
base potencial para uma atividade futura ou atual.

- Acompanhar novas tendências: o resgate humano e o destaque da História da Computação tem sido valorizados por instituições governamentais e iniciativas privadas em todo o mundo, por meio de homenagens, criação de museus, etc.

- Revalorizar o fator humano: toda tecnologia disponível foi desenvolvida por pessoas reais, cada uma acrescentando sua pequena ou grande contribuição. Sem a compreensão do fator humano da Computação, não é possível entender a trajetória histórica da área, nem o seu possível desenrolar futuro.

É possível ainda, acrescentar a possibilidade de atração de novos talentos para a Computação, motivados pelo fator inspirador que o ensino das histórias dos grandes feitos computacionais e personalidades podem promover.

\section{Trabalhos Relacionados}

Alguns recursos educacionais contemporâneos que vem sendo explorados por educadores como apoio no processo de ensino-aprendizagem de História da Computação são, principalmente, os museus virtuais e as linhas do tempo digitais.

Dalasso et al. (2014) apresentam um museu virtual 3D brasileiro sobre História da Computação, desenvolvido através da plataforma de mundos virtuais OpenSimulator com galerias que incluem temáticas como Gerações dos Computadores, Personalidades, Linguagens e Pré-História da Computação. Outra iniciativa nacional interessante é o Museu do Computador da Universidade Estadual de Maringá ${ }^{1}$ que possui um acervo online sobre personalidades e dispositivos. Alguns exemplos de museus virtuais de História da Computação internacionais são o Museu Virtual da Informática (MVI) [Almeida 2006] e o Computer History Museum, que apesar de ser um museu físico localizado em Boston, também disponibiliza um tour virtual pelo site do museu ${ }^{2} \mathrm{e}$ possui uma linha do tempo digital ${ }^{3}$ com marcos da história da Computação. Outras linhas do tempo digitais interessantes a serem destacadas são as do Centre of Computing History $^{4}$ e as do Museu da Computação e Informática (MCI $)^{5}$, que apresenta um acervo de linhas do tempo em diferentes temáticas.

Já na área de jogos sérios para o ensino de Computação, existem diversos trabalhos que utilizam jogos baseados em cartas, tais como: o jogo Simules-W [Monsalve et al. 2010] para o ensino de Engenharia de Software; o jogo Baralho das Variáveis para o ensino de princípios de programação [Kahwage et al. 2013]; o jogo JEES [Figueiredo et al. 2010] para o ensino de Gerência de Configuração, entre outros. Destaca-se também o trabalho de Curzon e McOwan (2008) para ensinar conteúdos de programação e interação humano-computador utilizando jogos e truques com baralhos. O livro clássico "Computer Science Unplugged" [Bell et al. 1998], que é constantemente atualizado e empregado no ensino, também utiliza cartas em diversas atividades lúdicas para o ensino de conceitos de Computação. Por fim, o Computing

http://www.din.uem.br/museu/museuvirtual.htm

http://www.computerhistory.org/

http://www.computerhistory.org/timeline/

http://www.computinghistory.org.uk/cgi/computing-timeline.pl

http://www.mci.org.br/ 
Research Association juntamente com o Anita Borg Institute disponibilizam uma página ${ }^{6}$ de relatos de educadores e pesquisadores sobre o uso de cartas no ensino de Computação muito interessante para consulta.

\section{Computasseia}

O Computasseia é um jogo de cartas de estratégia desenvolvido, prioritariamente, para dar suporte ao ensino da história da Computação em diferentes níveis de ensino (técnico, graduação e pós-graduação), tendo em vista que os conteúdos abordados e a linguagem trabalhada são abrangentes o suficiente para a inclusão de tal estratégia.

Este jogo foi elaborado como recurso educacional de apoio ao ensino de História da Computação pensando na dificuldade encontrada algumas vezes por educadores ao trabalharem de forma atrativa para os alunos tais temáticas históricas repletas de nomes, fatos e datas importantes. O jogo entra no processo de ensino-aprendizagem como ferramenta lúdica, auxiliando na aprendizagem e retenção de conteúdos pelos alunos.

O nome "Computasseia" foi escolhido para o jogo em referência ao poema grego clássico "Odisseia" de Homero, disponível em edição de língua portuguesa [Lourenço 2003], que narra a jornada do herói Odisseu (ou Ulisses para os romanos) na ilha de Ítaca, durante o final da guerra de Tróia. $\mathrm{O}$ nome foi escolhido não somente devido à importância histórica da obra para a Literatura Ocidental [Lopes 2013], mas também pelo seu conteúdo, mostrando a longa e desafiadora jornada do herói, aludindo assim ao caminho da Computação para firmar-se como ciência e da trajetória do jogador explorando este caminho.

O Computasseia é composto por um conjunto inicial de 60 cartas distintas, cada uma representando uma parte significativa da história da Computação. Cada carta do jogo pertence a uma Categoria e a uma ou mais Áreas do Conhecimento, a fim de melhor organizar os conteúdos e trazer mais um nível de informação ao jogador, respectivamente. As Categorias e Áreas do Conhecimento são descritas nas subseções a seguir.

O conjunto inicial de cartas do jogo pode ser estendido, para incluir novas cartas no futuro, seguindo a padronização do jogo. É possível, inclusive, desenvolver novos decks de cartas temáticos dentro de determinada subárea de uma Área do Conhecimento, para que a temática escolhida possa ser trabalhada de forma mais aprofundada. Todas as cartas e demais informações sobre o jogo Computasseia estão disponíveis no site do jogo ${ }^{7}$.

\subsection{As cartas do jogo}

\subsubsection{Categorias das cartas}

Cada carta em Computasseia é categorizada individualmente em uma das seguintes três categorias:

- Personalidades: representam pessoas que contribuíram de forma significativa com teorias, invenções, ideias ou princípios para pelo menos uma das Áreas do

http://www.cs.duke.edu/csed/wikipedia/cardsusing.html

http://gameinclass.ic.ufmt.br/computassy/ 
Conhecimento definidas na seção 4.1.2.

- Instituições: representam empresas, associações, sociedades e outras instituições com contribuições significativas para pelo menos uma das Áreas do Conhecimento definidas na seção 4.1.2.

- Eventos: representam acontecimentos em geral, tais como invenções, descobertas, reuniões, seminários, entre outros associados a, pelo menos, uma das Áreas do Conhecimento definidas na seção 4.1.2.

As Categorias podem ser observadas na parte superior da visão da frente das cartas (ver item 4 na Figura 1). A Figura 2 apresenta um exemplo de carta do jogo de cada uma das três categorias citadas.

\section{Frente}

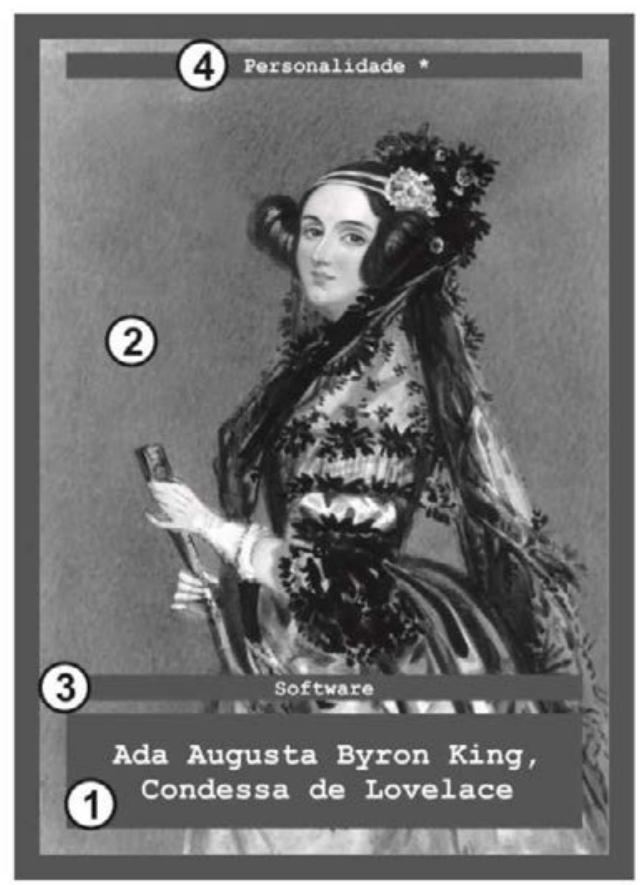

\section{Verso}

\section{0}

Ada Lovelace (1815-1852) foi

uma matemática e escritora inglesa reconhecida por ter escrito o primeiro algoritmo para ser processado por uma máquina, a máquina analitica de Charles Babbage. Ela desenvolveu algoritmos que permitiriam à máquina computar funçōes matemáticas, além de publicar uma série de notas sobre a máquina analítica. Assim, é considerada a primeira programadora da história.

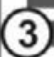

Ada Augusta Byron King,

1) Condessa de Lovelace

Figura 1. Exemplo de carta do jogo Computasseia e seus elementos indicados

\subsection{2. Áreas do Conhecimento}

Cada carta em Computasseia é classificada em uma ou mais Áreas do Conhecimento, definidas para melhor organização das cartas do jogo e para incrementar a carta com mais um nível de informação para o jogador. As áreas do Conhecimento foram selecionadas a partir da análise das subáreas da computação por AMC e IEEE (2013) e da organização das comissões especiais da Sociedade Brasileira de Computação ${ }^{8}$.

As Áreas do Conhecimento do jogo Computasseia são:

- Hardware: Compreende as tecnologias de desenvolvimento de equipamentos, materiais e demais tecnologias físicas que impactam a Computação. Exemplo: A

\footnotetext{
$8 \quad$ http://sbc.org.br/403-comissoes-especiais
} 
invenção dos transístores.

- Software: Compreende avanços de tecnologias e teorias de sistemas relacionados ao processo de desenvolvimento de software. Exemplo: Modelos de Análise de Cooper.

- Teoria Computacional e Matemática: Avanços teóricos na Computação e em áreas matemáticas de grande influência. Exemplo: Teoria dos Grafos.

- Computação e Sociedade: Compreende impactos sociais, culturais e legais causados, alterados ou acelerados pela adoção de novas tecnologias, bem como o contrário, em que particularidades de uma sociedade definiram os rumos da Computação. Exemplos: Primavera Árabe e o Marco Civil da Internet Brasileira.

- Gestão de Dados: Compreende os impactos de tecnologias e teorias para gestão de dados e seus processos de armazenamento, transmissão e visualização. Exemplo: A invenção do IBM 350, primeiro disco rígido comercializado.

As áreas designadas são correlacionadas e não se restringem apenas aos casos citados. As cartas podem ser encaixadas em mais de uma Área do Conhecimento, e.g. as contribuições de Alan Turing (1912-1954) podem se encaixar nas categorias de Hardware, Software e Teoria Computacional e Matemática. As Áreas do Conhecimento podem ser observadas na parte inferior da visão da frente e de verso das cartas (ver item 3 na Figura 1). A Figura 2 apresenta exemplos de carta do jogo de cada uma das áreas.
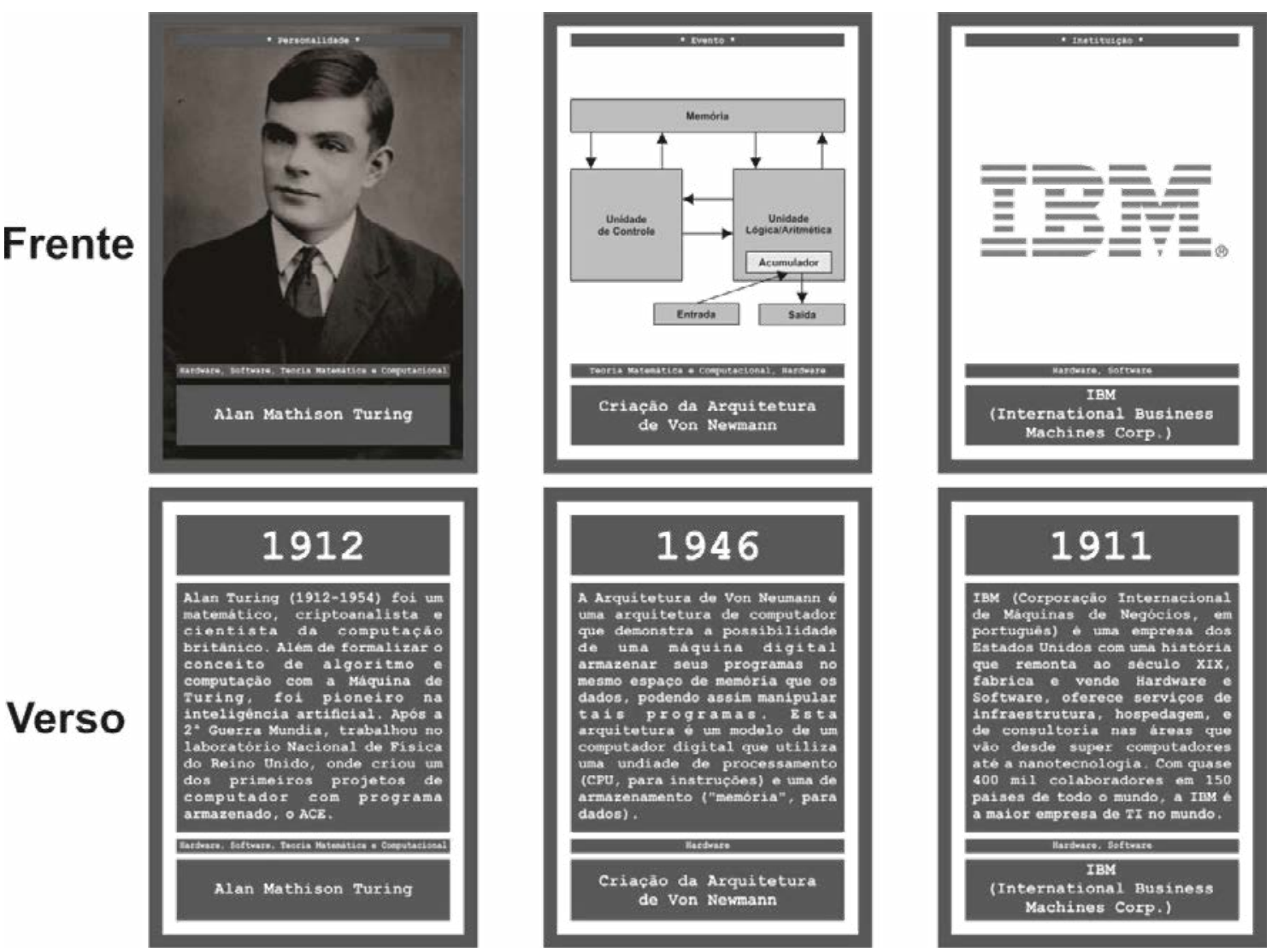

Figura 2. Exemplos de cartas do jogo Computasseia das Categorias Personalidade, Evento e Instituição.
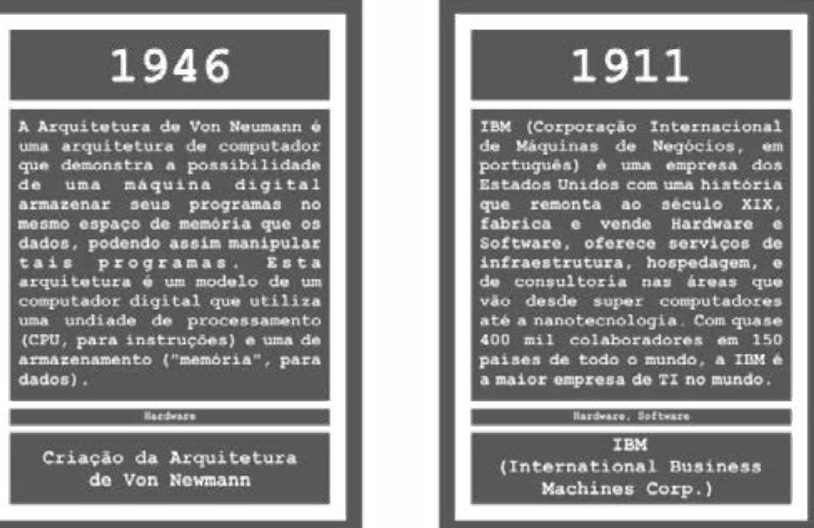


\subsubsection{Elementos das cartas e sua organização}

As cartas em Computasseia são compostas dos seguintes elementos gráficos que podem ser visualizados na Figura 1:

- Título (1): O nome da carta, indicando de forma direta o que a carta representa conforme sua Categoria: para Personalidades, o nome da pessoa representada (também pode conter um apelido ou epíteto se necessário); para Instituições, o nome e a sigla da instituição (se existir); e para Eventos, o nome como o acontecimento é popularmente conhecido.

- Imagem ilustrativa (2): Representação imagética da Personalidade, Instituição ou Evento da carta. Esse elemento encontra-se apenas na visão da frente da carta.

- Área do Conhecimento (3): Representada por texto, indicando a área ou áreas conforme a subseção 4.1.2.

- Categoria (4): Representada por texto, indicando a categoria da carta conforme a subseção 4.1.1. Esse elemento encontra-se apenas na visão da frente da carta.

- Descrição (5): Descrição textual que introduz o conceito da carta, complementando a informação do título e expandindo sua contextualização histórica e relevância computacional. Esse elemento encontra-se apenas na visão do verso da carta.

- Data (6): Marco temporal que está associado ao fato histórico que a carta representa, de acordo com a sua Categoria: para Personalidades, a data representa o ano de nascimento da pessoa; para Instituições, o ano de sua fundação; e para os eventos, a data exata ou aproximada em que foi realizado. Esse elemento encontra-se apenas na visão do verso da carta.

\subsection{Dinâmica do jogo}

A dinâmica do jogo Computasseia consiste na elaboração conjunta pelos jogadores de uma Linha do Tempo de marcos da história da Computação, formada pela disposição sequencial em ordem crescente da Data das cartas, constituindo uma cronologia. Essa dinâmica é inspirada no jogo infanto-juvenil Timeline ${ }^{9}$ da Galapagos Jogos.

Cada jogador recebe no início do jogo um conjunto de 6 cartas atribuídas de forma aleatória. As demais cartas restantes do jogo são empilhadas em um montante denominado Montante de Compra com a visão da frente voltada para cima. O Computasseia comporta de 2 a 6 jogadores devido à sua quantidade de cartas atual.

Ao receber as cartas, o jogador que as recebe deve continuar observando suas cartas apenas a partir da visão frontal das mesmas, sem observar o lado das cartas que contém a Data e a Descrição.

Os jogadores se posicionam em volta do local em que será montada a Linha do Tempo, preferencialmente sobre uma mesa. Um por um, em sentido horário ou antihorário (a ser definido pelos jogadores), o jogador da vez deverá escolher uma das cartas em sua mão para utilizar em sua jogada. Assim que fizer a escolha, deverá tentar inseri-

9 https://www.galapagosjogos.com.br/jogos/timeline 
la na posição que julga ser a correta na Linha do Tempo. Feito isto, a face da carta jogada é virada, revelando suas informações de Data e Descrição contidas no verso. Logo, deve ser verificado o acerto ou erro da jogada.

Caso o jogador tenha errado a posição da carta, essa carta é mantida na mesa, porém a mesma deve ser recolocada na sua posição apropriada na Linha do Tempo e o jogador deve retirar uma nova carta no Montante de Compra para as suas cartas da mão. A vez de jogada é passada ao próximo jogador.

O jogo acaba quando um dos jogadores consegue descartar de forma apropriada todas as cartas de sua mão para a Linha do Tempo do jogo, sendo o mesmo declarado vencedor da partida, ou quando o Montante de Compra se esgota (neste caso, o vencedor da partida é o jogador (ou jogadores) com o menor número de cartas em mão).

\subsubsection{Exemplos de jogadas}

A Figura 3 ilustra uma mesa com um grupo de jogadores jogando uma partida de Computasseia. O Jogador 1 é escolhido dentre os participantes da mesa para fazer a distribuição das cartas. O Jogador 1 distribui as cartas de modo que nenhum jogador (incluindo ele mesmo) possa observar a visão do verso das cartas. A área $\boldsymbol{a}$ ) da Figura 3 mostra a distribuição de cartas já realizada e sua disposição em relação aos jogadores.

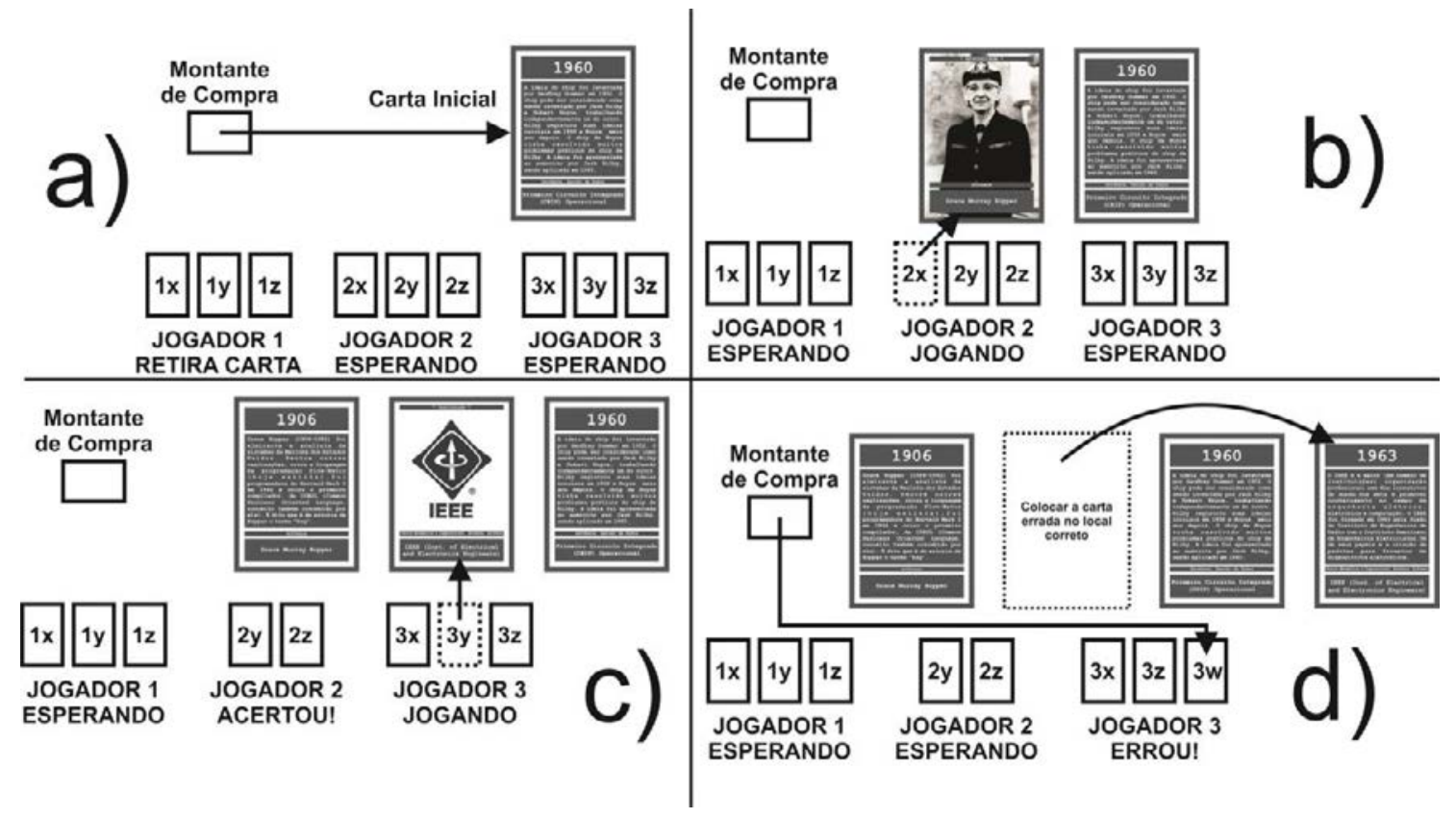

Figura 3. Passos de uma demonstração de jogada

Em seguida o Jogador 1, responsável pela distribuição das cartas, retira uma carta do Montante de Compra para dar início ao jogo, colocando esta Carta Inicial na Linha do Tempo com seu verso virado para cima, exibindo sua Data e Descrição. Isso é feito de maneira a nenhum jogador iniciar o jogo com vantagem, pois a carta inicial sempre estará na posição correta.

Em seguida, o Jogador 2 (o próximo jogador da sequência) será o primeiro a realizar uma jogada de fato. O Jogador 2 escolhe a carta $2 \boldsymbol{x}$ e resolve incluí-la no lado esquerdo da Carta Inicial, como pode ser observado na área $\boldsymbol{b}$ ) da Figura 3. Depois que 
ele fez este movimento, o próprio jogador vira a carta e revela para todos a Data e a Descrição da carta. É possível observar na área c) da Figura 3 que o Jogador 2 acertou sua jogada. Assim, sua carta permanece na posição correta e o mesmo possui uma carta a menos em mãos, ou seja, está mais próximo da vitória.

Agora, é a vez do Jogador 3 fazer sua jogada. O jogador 3 escolhe a carta $3 \boldsymbol{y}$ e decide por colocá-la entre as duas cartas já dispostas na Linha do Tempo. Ao virar a carta a fim de revelar a Data, conforme ilustrado na Figura 3 área $\boldsymbol{d}$ ), é possível notar que ele errou a posição correta temporal dos acontecimentos. A carta jogada é, por sua vez, reposicionada na posição correta na Linha do Tempo, e o Jogador 3 compra uma nova carta do Montante de Compra. A vez do jogo é passada para o Jogador 1.

Devido ao seu erro, o Jogador 3 está com o mesmo número de cartas, ou seja, mais longe da vitória do que o Jogador 2 que acertou na sua vez de jogar. Conforme a Linha do Tempo cresce, a dificuldade do jogo aumenta, tendo em vista que o risco de erro é maior devido ao número de locais que os jogadores podem escolher para introduzir a carta. O jogo segue até que um dos jogadores fique sem cartas na mão (esse jogador é declarado o vencedor da partida) ou quando o Montante de Compra se esgota.

\section{Conclusões}

Este trabalho apresentou um jogo sério para o ensino de História da Computação chamado Computasseia que aborda fatos importantes de diferentes áreas do conhecimento da Computação e que pode ser utilizado em diferentes níveis de ensino. A sua dinâmica estratégica estimula o jogador na retenção dos conteúdos das cartas e a ludicidade do jogo incentiva o seu envolvimento com a disciplina. Os autores do presente trabalho acreditam que o jogo de cartas proposto é um recurso acessível aos educadores (podendo ser facilmente impresso no site do jogo e utilizado) e pretendem estender o jogo no futuro adicionando novos elementos de estratégia e criando novos decks de cartas temáticas de subáreas específicas para incrementar o jogo atual.

\section{Referências}

ACM e IEEE (2013) "Computer Science Curricula 2013: Curriculum Guidelines for Undergraduate Degree Programs in Computer Science”, ACM, New York, 518 p.

Baptista, R., e Carvalho, C. V. D. (2013) "TimeMesh-a serious game for european citizenship", EAI Endorsed Transactions on Game Based Learning, 13(01-12), p. 1-7.

Bell, T. C., Witten, I. H., Fellows, M. (1998) “Computer Science Unplugged: Off-line activities and games for all ages", Computer Science Unplugged.

Berry, G., Sheard, J., Quartly, M. (2011) “A Virtual Museum of Computing History: an educational resource bringing the relationship between people and computers to life", In: Proceedings of the Thirteenth Australasian Computing Education Conference-Volume 114, Australian Computer Society, Inc., p. 79-86.

Curzon, P. e McOwan P.W. (2008) "Engaging with Computer Science through Magic Shows", In: ACM. ITiCSE 2008, The 13th Annual Conference on Innovation and Technology in Computer Science Education, p.179-183.

Dalsasso, P. Bos, A. S., Bernardi, G. Müller, F. M. Cordenonsi, A. Z. (2014) 
"Resgatando A História da Computação através de um Museu Virtual 3D Interativo", In: XXII Workshop sobre Educação em Informática (WEI 2014), Brasília, DF.

Figueiredo, K., Ferreira, J., Murta, L., Clua, E. (2010) “Jogo de Estratégia de Gerência de Configuração", In: III Fórum em Educação de Engenharia de Software (FEES), Simpósio Brasileiro de Engenharia de Software (SBES), Salvador.

Fonseca Filho, C. (2007) "História da computação: O Caminho do Pensamento e da Tecnologia”, EDIPUCRS, Porto Alegre, 205 p.

Froschauer, J., Arends, M., Goldfarb, D., Merkl, D. (2011) "Towards an online multiplayer serious game providing a joyful experience in learning art history", In: Games and Virtual Worlds for Serious Applications, IEEE Conference, p. 160-163.

Haydn, T., Stephen, A., Arthur, J., Hunt, M. (2014) "Learning to teach history in the secondary school: A companion to school experience", Routledge, New York, 360 p.

Kahwage, C., de França, E. , Nunes, R. , Carvalho, R., Souza, D. (2013) "Jogo Baralho das Variáveis”, In: XXI Workshop sobre Educação em Informática (WEI 2013).

Krassmann, A. L., Falcade, A., Silva, L. E. G., Medina, R. D. (2015) "Serious Games to Computer Networks Learning With CyberCIEGE: A Case Study in Brazilian Higher Education", In: XXIII Workshop sobre Educação em Informática (WEI 2015).

Leite, L. S., Sibaldo, M. A. A., Carvalho, T. B. A., Souza, R. (2014) "Montanha de Chomsky: jogo tutor para auxílio no ensino de Teoria da Computação", In: XXII Workshop sobre Educação em Informática (WEI 2014), Brasília, DF.

Lopes, J. J. (2013). "A Ilíada e a Odisseia - Dois Pilares da Civilização Grega e Legado Para a Posteridade”, In: Revista Memento, 4(1), p. 118-127.

Lourenço, F. (2003) "Homero, Odisseia”. Lisboa, Livros Cotovia.

Michael, D. R., e Chen, S. L. (2005) "Serious games: Games that educate, train, and inform", Muska \& Lipman/Premier-Trade.

Monsalve, E., Werneck, V., Leite, J. C. S. P. (2010) "Simules-w: Um jogo para o ensino de engenharia de software", In: III Fórum em Educação de Engenharia de Software (FEES), Simpósio Brasileiro de Engenharia de Software (SBES), Salvador, p. 17-26.

Neves, I. B. D. C. (2011). "Jogos digitais e potencialidades para o ensino de história: um estudo de caso sobre o history game Tríade-liberdade, igualdade e fraternidade", Dissertações de Mestrado, Programa de Pós-Graduação em Educação (PPGE), Universidade Federal da Bahia, 243 p.

Nunes, I. F. e Parreira Júnior, P. A. (2015) "RPG4Sorting - Um Jogo Educacional para Auxílio ao Ensino de Métodos de Ordenação", In: XXIII Workshop sobre Educação em Informática (WEI 2015), Recife, PE.

Vahldick, A., Mendes, A. J., Marcelino, M. J., Hogenn, M., Schoeffel, P. (2015) "Testando a Diversão em um Jogo Sério para o Aprendizado Introdutório de Programação”, In: XXIII Workshop sobre Educação em Informática (WEI 2015). 